\title{
Genome-wide mapping of the dominance effects based on breed ancestry for semen traits in admixed Swiss Fleckvieh bulls
}

\author{
N. Khayatzadeh, ${ }^{1 *}$ G. Mészáros, ${ }^{1}$ Y. T. Utsunomiya, ${ }^{2}$ F. Schmitz-Hsu, ${ }^{3}$ F. Seefried, ${ }^{4}$ U. Schnyder,${ }^{4}$ \\ M. Ferenčaković, ${ }^{5}$ J. F. Garcia, ${ }^{2,6}$ I. Curik, ${ }^{5}$ and J. Sölkner ${ }^{1}$ \\ ${ }^{1}$ Division of Livestock Sciences, Department of Sustainable Agricultural Systems, University of Natural Resources \\ and Life Sciences Vienna (BOKU), Gregor-Mendel-Strasse 33, A-1180 Vienna, Austria \\ ${ }^{2}$ Departamento de Medicina Veterinária Preventiva e Reprodução Animal, Faculdade de Cinêcias Agrárias Veterinárias, \\ UNESP-Univ Estadual Paulista, Araçatuba, São Paulo, Brazil 16050-680 \\ ${ }^{3}$ Swissgenetics, Meielenfeldweg 12, Postfach, 3052 Zollikofen, Switzerland \\ ${ }^{4}$ Qualitas AG, Chamerstrasse 56, Ch-6300, Zug, Switzerland \\ ${ }^{5}$ Department of Animal Science, Faculty of Agriculture, University of Zagreb, Svetošimunska cesta 25, 10000 Zagreb, Croatia \\ ${ }^{6}$ Departamento de Apoio, Saúde e Produção Animal, Faculdade de Medicina Veterinária de Araçatuba, UNESP-Univ Estadual Paulista, \\ Araçatuba, São Paulo, Brazil 16050-680
}

\section{ABSTRACT}

Heterosis is the beneficial deviation of crossbred progeny from the average of parental lines for a particular trait. Heterosis is due to nonadditive genetic effects with dominance and epistatic components. Recent advances in genotyping technology have encouraged researchers to estimate and scan heterosis components for a range of traits in crossbred populations, applying various definitions of such components. In this study, we defined the intralocus (dominance) component of heterosis using local genetic ancestry and performed genome-wide association analysis for admixed Swiss Fleckvieh bulls and their parental populations, Red Holstein Friesian and Swiss Simmental, for semen traits. A linear mixed model for 41,824 SNP, including SNP additive genetic, breed additive, and breed dominance effects on 1,178 bulls (148 Red Holstein Friesian, 213 Swiss Simmental, and 817 Swiss Fleckvieh) with a total of 43,782 measurements was performed. In total, 19 significant regions for breed dominance were identified for volume (2 regions on Bos taurus autosome 10 and 22 ) and percentage of live spermatozoa (17 regions on Bos taurus autosome 3, 4, 5, 7, 13, 14, and 17), and genes associated with spermatogenesis, sperm motility, and male fertility traits were located there. No significant region for breed dominance was detected for total number of spermatozoa. The signals for breed dominance were relatively wide, most likely due to limited numbers of recombination events in a small number of

Received May 3, 2019.

Accepted August 1, 2019.

*Corresponding author: negar.khayatzadeh@boku.ac.at generations (10-15 generations) of crossbreeding in the recent Swiss Fleckvieh composite.

Key words: breed dominance, dominance component of heterosis, local genetic ancestry, genome-wide mapping

\section{INTRODUCTION}

The crossing of inbred lines to produce hybrids plays an important role in the framework of genetic improvement in plant and livestock breeding (Falconer and Mackay, 1996). Crossbreeding is used to design an optimized mating system to benefit from the breed complementarity of additive genetic effects of parental breeds, decreased inbreeding levels, and inbreeding depression and to make use of a desirable level of nonadditive genetic effects of heterosis (Gregory et al., 1966; Cundiff, 1970). Heterosis is a natural phenomenon in which hybrid progenies present more vigorous performance, especially for health and fitness traits, compared with their mid-purebred parents (Shull, 1908). Heterosis is the opposite of inbreeding depression in the sense that the vigor lost in pure inbred lines can be restored in crossbreeding between pure lines (Falconer and Mackay, 1996).

In dairy cattle, heterosis is more pronounced in polygenic traits with relatively low heritability, such as fertility, health traits, and calving performance, which are influenced more by nonadditive genetic effects than by additive genetic effects. Global heterosis for production traits in dairy cattle is reported as 3 to $4 \%$, whereas higher levels (5\%) are observed for functional and reproductive traits (Freyer et al., 2008; Kargo et al., 2012). The amount of heterosis depends on the type of crossbreeding mating system, allele frequency difference between parental populations, and degree 
and direction of dominance at each locus (Falconer and Mackay, 1996). The genetic basis of heterosis consists of a favorable (intralocus) dominance component, where the heterozygous combinations imply partial to overdominance effects rather than both homozygous combinations (Shull, 1908), and an (interloci) epistatic loss component, as the result of gene interaction between loci (Dickerson, 1973; Hill, 1982; Kinghorn, 1983).

Owing to recent advances in genotyping technology and designing various SNP chips, which provide enough information at the genome-wide level, several studies have used genomic markers to estimate genomic heterosis and its components in livestock. In some studies, average genome-wide heterozygosity, retained heterozygosity (Dickerson, 1973) as the genomic breed fraction of each contributing parental breed, derived from Admixture (Alexander et al., 2009), and deviation of adjusted crossbred phenotypes from the mid-parent were used to define breed heterozygosity for carcass and growth traits in Canadian beef cattle (Akanno et al., 2017, 2018a) and female fertility traits in beef crossbred heifers (Basarab et al., 2018). Khayatzadeh et al. (2018b) also estimated genomic heterosis components (breed dominance and epistatic loss) using local genetic ancestry proportions, estimated at the SNP level, for semen traits [volume $(\mathrm{mL})$, concentration (count $\times$ $10^{9} / \mathrm{mL}$ ), total number of spermatozoa, and percentage of live spermatozoa] in admixed Swiss Fleckvieh bulls compared with their parental populations, Red Holstein Friesian (RHF) and Swiss Simmental (SI). In a subsequent study, Khayatzadeh et al. (2018a) performed SNP genome-wide mapping for breed dominance components of heterosis for percentage of live spermatozoa in Swiss Fleckvieh bulls. In another study, Akanno et al. (2018b) applied a straight genome-wide association study model to estimate dominance and epistatic interaction for growth traits in Canadian beef cattle.

The objective of the current study was to map breed dominance components of heterosis for semen traits, including volume, transformed number of spermatozoa (TNS), and percentage of live spermatozoa (\%LS). Coding was based on local genetic ancestries, extracted from the local admixture, estimated by LAMP software (Sankararaman et al., 2008), and genomic regions were identified by associating breed dominance with these semen traits in admixed Swiss Fleckvieh bulls. We excluded sperm concentration because no significant heterosis effect was identified for this trait in a previous study (Khayatzadeh et al., 2018b). The breed dominance component of heterosis was estimated to be significant and was $1.24 \mathrm{~mL}$ for volume, $0.28 \times 10^{9}$ for TNS, and $1.40 \%$ for $\% \mathrm{LS}$.

\section{MATERIALS AND METHODS}

\section{Phenotypic Records, Herd, and Management Description}

Swiss Fleckvieh is a recent composite cattle breed that has been established over the last $40 \mathrm{yr}$ in Switzerland with the aim of combining the strengths of the 2 originating breeds, RHF and SI. The Swiss Fleckvieh breeding program focuses on high milk yield derived from RHF ancestry as well as functional and fitness traits of the SI breed.

Animals with a pedigree-based RHF blood share of 0.125 to 0.875 are registered formally as Swiss Fleckvieh if RHF blood shares of both parents and all 4 grandparents are also within those limits. Animals with $<0.125 \mathrm{RHF}$ are considered Simmental, and those with $>0.875$ RHF are categorized as RHF in Swissherdbook (Zollikofen, Switzerland). In this study, this formal definition was not followed, and all of the bulls with a range of 0.01 to 0.99 RHF blood proportion as admixed bulls were considered.

Phenotypic records on semen traits were made available by Swissgenetics from the Mülligen AI station in Switzerland. Bulls are generally kept in tiestalls. Semen is collected twice a week; a teaser bull is used to prepare bulls, and then semen is collected with a dummy and artificial vagina. In this AI station, ejaculates are collected 1 or 2 times per day for the same bull. Records from 2000 to 2015 were considered. The routinely recorded traits for each ejaculate were volume $(\mathrm{mL})$, concentration (count $\left.\times 10^{9} / \mathrm{mL}\right)$, and \%LS. Total number of spermatozoa for each ejaculate was calculated by multiplying volume $(\mathrm{mL})$ by concentration. Percentage of live spermatozoa was determined by visual assessment. In total, 68,475 records were received for 1,298 bulls (171 RHF, $226 \mathrm{SI}$, and 901 admixed Swiss Fleckvieh bulls) born between 1990 and 2014. Bulls with at least 10 records were kept for analysis. Ejaculate volumes in the range of 1 to $25 \mathrm{~mL}$ were kept for analysis. Ejaculates with an interval of less than 3 d since recent ejaculation were removed. Because total number of spermatozoa was not normally distributed, the following transformation was performed (Box and Cox, 1964):

$$
\mathrm{TNS}=\left(\text { total number of spermatozoa }{ }^{0.3}-1\right) / 0.3 .
$$

Observations on TNS $\left(\times 10^{9}\right)$ and \%LS beyond the range mean \pm 3 standard deviations were discarded. Summary statistics on semen traits are presented in Table 1. 
Table 1. Summary statistics (mean \pm SD) of semen traits (volume, transformed number of spermatozoa, and percentage of live spermatozoa)

\begin{tabular}{|c|c|c|c|c|c|}
\hline Trait & Records (no.) & Bulls (no.) & \multicolumn{3}{|c|}{ Breed $^{1}$} \\
\hline Volume $(\mathrm{mL})$ & 42,830 & 1,177 & $5.63 \pm 2.67$ & $5.32 \pm 2.35$ & $5.61 \pm 2.50$ \\
\hline Live spermatozoa (\%) & 42,121 & 1,169 & $85.66 \pm 3.10$ & $86.11 \pm 3.89$ & $86.42 \pm 3.28$ \\
\hline
\end{tabular}

${ }^{1}$ RHF = Red Holstein Friesian; SI = Simmental; SF = Swiss Fleckvieh.

${ }^{2}$ Transformed number of spermatozoa.

\section{Genotype Data}

A subset of 44,999 SNP for 1,411 bulls, genotyped with Illumina Bovine SNP 50k, 150k, and 777k BeadChips (Illumina, San Diego, CA), was used. The standard quality control was performed with PLINK 2.0 (Purcell et al., 2007; Chang et al., 2015) to remove from the data set SNP with minor allele frequency lower than 0.01 and call rate less than 0.95 and those deviating from Hardy-Weinberg equilibrium with $P<$ $10^{-6}$ (Fisher's exact test). Finally, 41,824 SNP for 1,178 (148 RHF, $213 \mathrm{SI}$, and 817 admixed) bulls remained for further analyses.

\section{Prediction of Local Ancestries}

The LAMP 2.5 (Sankararaman et al., 2008) program was used in ancestry mode to infer locus-specific ancestry proportions at each SNP position along the genome of admixed bulls. The LAMP program applies a hidden Markov model clustering algorithm to infer locus-specific ancestries, computing the ancestry structure for overlapping windows of contiguous SNP. Configurations for LAMP were as in our previous studies (Khayatzadeh et al., 2018a,b). Ancestry origin of each SNP was estimated for each admixed bull with respect to the allele frequency of 2 pure ancestral populations, representing $0,0.5$, and 1 as the ancestry proportions for each SNP. The 0 and 1 values indicate that 2 alleles at each single locus have the same breed origin, and 0.5 indicates that each allele originates from a different ancestral population at the corresponding locus. In our case, pure SI ancestry was coded as 0 and pure RHF ancestry was coded as 1 .

\section{Estimation of SNP Additive, Breed Additive, and Breed Dominance Effects}

Genome-wide analyses for autosomal SNP were performed for volume $(\mathrm{mL})$, TNS $\left(\times 10^{9}\right)$, and \%LS in Swiss Fleckvieh bulls as well as bulls of their parental purebred populations (RHF and SI). A single SNP genome-wide model was run, including SNP additive genetic, breed additive, and breed dominance effects, derived from LAMP, as regression coefficients. The analyses were performed using the MIXED procedure and maximum likelihood method in SAS (SAS Institute Inc., 2009) for the following model:

$$
\mathbf{y}=1 \mu+\mathbf{X} \mathbf{b}+\mathbf{Z a}+\mathbf{s} \beta+\mathbf{v} \alpha+\mathbf{w} \delta+\mathbf{e},
$$

where $\mathbf{y}$ is the vector of observations for volume $(\mathrm{mL})$, TNS $\left(\times 10^{9}\right)$, and \%LS; $\mu$ is the population mean; $\mathbf{X}$ and $\mathbf{Z}$ are the design matrices for fixed effects and random effect of animal, considering repeated measurements, respectively; $\mathbf{b}$ is the vector of fixed effects of age $(<16,16-72$, and $>72 \mathrm{mo})$, year and season (February-May, June-September, and October-January), ejaculate intervals $(3-6,7-9$, and $>9 \mathrm{~d})$, and semen collector; $\mathbf{a}$ is the vector of random effects of animal with $N \sim\left(0, \mathbf{I} \sigma_{a}^{2}\right) ; \mathbf{I}$ is an identity matrix, $\mathbf{s}$ is a vector of SNP genotypes, coded as 0,1 , and 2 for the number of a particular allele at the SNP; $\beta$ is the additive effect of that SNP allele; $\mathbf{v}$ is a vector of locus-wise ancestry proportions of RHF, coded as $0,0.5$, and 1 (pure SI were therefore coded as 0 and pure RHF were coded as 1 for all SNP); $\alpha$ is the regression coefficient for breed additive effect based on these ancestry proportions; $\mathbf{w}$ is a vector of 0 and 1 values ( 0 when both alleles of the locus were derived from the same breed and 1 when the 2 alleles originated from different ancestries; codes were 0 for all purebred bulls); $\delta$ is the regression coefficient for the breed dominance effect; and $\mathbf{e}$ is the vector of residual effect with $N \sim\left(0, \mathbf{I} \sigma_{e}^{2}\right)$, where $\sigma_{a}^{2}$ and $\sigma_{e}^{2}$ are variances due to animal and residual effects, respectively.

Note that the effects of animal were included to account for repeated measurements on the same individual without considering their covariation through a genomic relationship matrix. No other correction for population stratification was performed. This was done because crossbred populations are stratified by breed ancestry and heterosis is due to differences in ancestry, particularly of crossbred and purebred animals. Therefore, performing correction of population stratification 
for the total population of purebred and admixed animals would interfere with the search of signals of heterosis. Preliminary analyses showed this very clearly.

To perform genome-wide correction for multiple hypothesis testing, a $-\log P$-value of 6 was used to indicate significance threshold. The actual Bonferroni threshold for the 41,824 SNP was 5.92. The threshold is conservative, particularly for the breed additive and breed dominance effects, considering the small number of recombination events and the resulting small number of independent segments along the genome, considering the period since the start of crossbreeding (around 10 generations ago).

Because the significant signals of heterosis covered large intervals of SNP, we identified the significant regions by the location of the first and last flanking SNP with a significant $P$-value of $<10^{-6}$. For the single significant SNP, belonging to a peak close to the threshold line, the genomic regions related to heterosis were identified $\pm 1 \mathrm{Mb}$ around the single significant SNP.

\section{Gene Annotation}

The significant regions were analyzed to search for genes associated with male fertility and semen quantity and quality traits. Only genes with known function in Entrez (https://www.ncbi.nlm.nih.gov), UniProt (https://www.uniprot.org), or GeneCard (https://www .genecards.org) were reported.

\section{RESULTS}

\section{Genetic Effects in the Model}

The genetic factors in the model were SNP additive, breed additive, and breed dominance effects. This model was built based on the model for inbreeding mapping for semen traits in Austrian Fleckvieh bulls suggested by Ferenčaković et al. (2017). Figure 1 provides Manhattan plots for these 3 effects using chromosome 14 as a sample reference to inspect the patterns of $P$-values. For plots of all autosomal chromosomes, see Supplemental Figure S1 (https://doi.org/10.3168/jds .2019-16899). The Manhattan plot for SNP additive effects (Figure 1) is typical for this type of analysis, with much variation even for neighboring SNP. The plots for breed additive and breed dominance effects (Figures 1) are much more patterned, mostly because the ancestry of any particular segment of a chromosome changes based only on recombination events, which are typi-
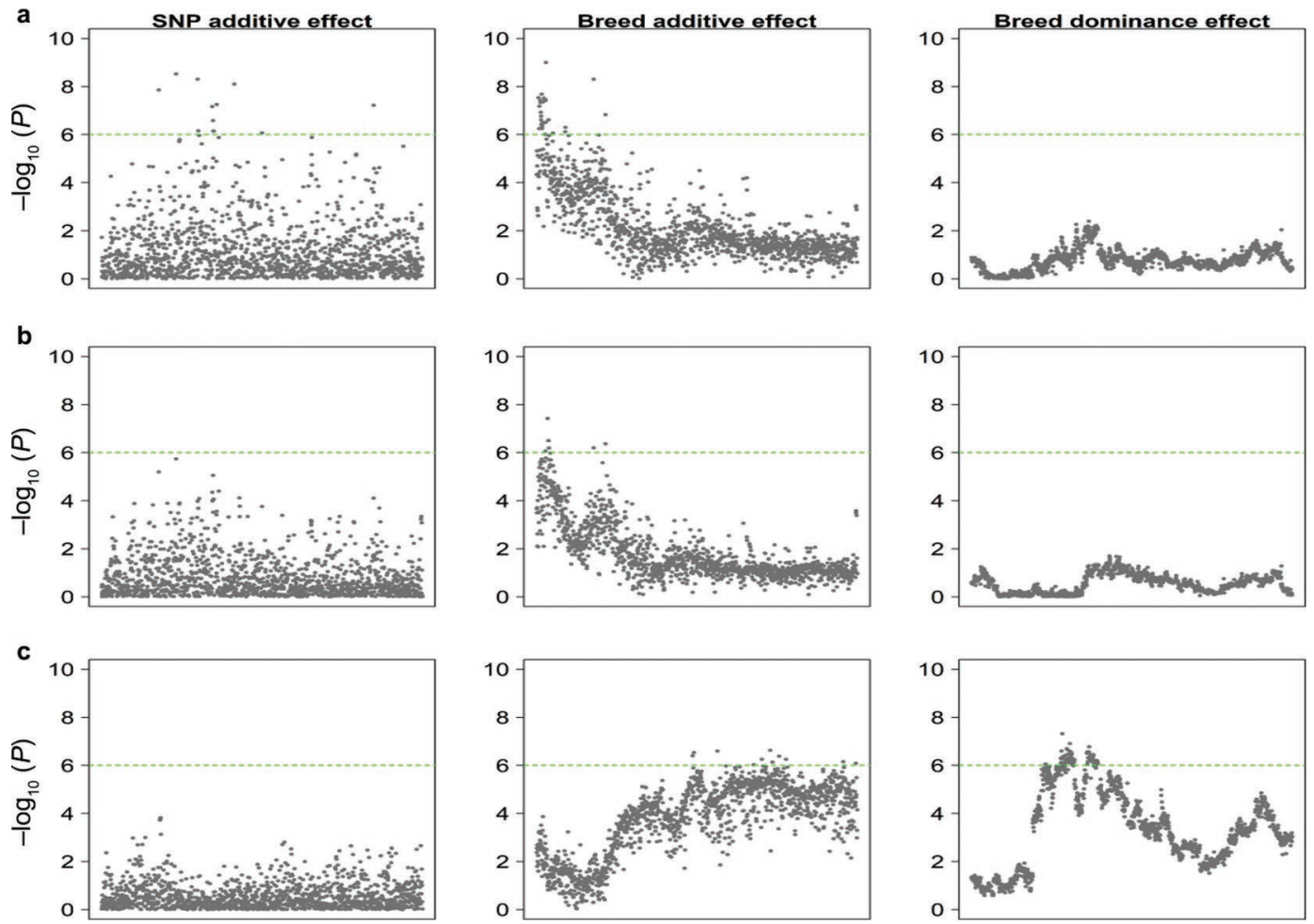

Figure 1. Chromosome-wise mapping of SNP additive genetic, breed additive, and breed dominance effects on BTA14 for (a) volume (mL), (b) transformed number of spermatozoa $\left(\times 10^{9} / \mathrm{mL}\right)$, and (c) live spermatozoa (\%). The horizontal dotted line indicates a $-\log P$-value of 6 as the significance threshold. 
cally not more than 1 or 2 per meiosis per chromosome. Therefore, neighboring SNP are likely to show the same ancestry pattern.

\section{Genome-Wide Mapping of Breed Dominance Effects}

For this study, we explored genomic regions showing significant signals for breed dominance, the key component of heterosis. In total, 19 regions [2 regions for volume $(\mathrm{mL})$ and 17 regions for \%LS] were identified. The length of the significant regions, except the single significant SNP, ranged from $71 \mathrm{~Kb}$ on BTA7 (47.31-47.39 $\mathrm{Mb})$ to $10.42 \mathrm{Mb}(63.09-73.52 \mathrm{Mb})$ on BTA13. The average length of the regions was $3.33 \pm$
$2.96 \mathrm{Mb}$, with $37.50 \pm 42.14 \mathrm{SNP}$ spanning the identified regions (Figures 2 and 3 ).

On BTA10 for volume (mL), only 1 SNP (40.61 Mb) passed the threshold. A region on BTA22 (41.78-44.77 $\mathrm{Mb})$ was also identified for volume $(\mathrm{mL})$, with 22 genes. Significant genomic regions for \%LS were identified on BTA3, 4, 5, 7, 12, 13, 14, and 17 .

Three significant heterosis signals (9.34-16.45, 24.5624.75, and 59.35-62.44 Mb) on BTA3 with 1 significant SNP (119.07 $\mathrm{Mb})$ and 2 significant signals on BTA4 (77.61-78.98 and $82.38-88.44 \mathrm{Mb}$ ) were identified. Two significant regions on BTA5 (28.85-30.19 and 113.43$120.75 \mathrm{Mb}$ ) were found to be associated with heterosis. Two close heterosis signals were also detected on BTA7

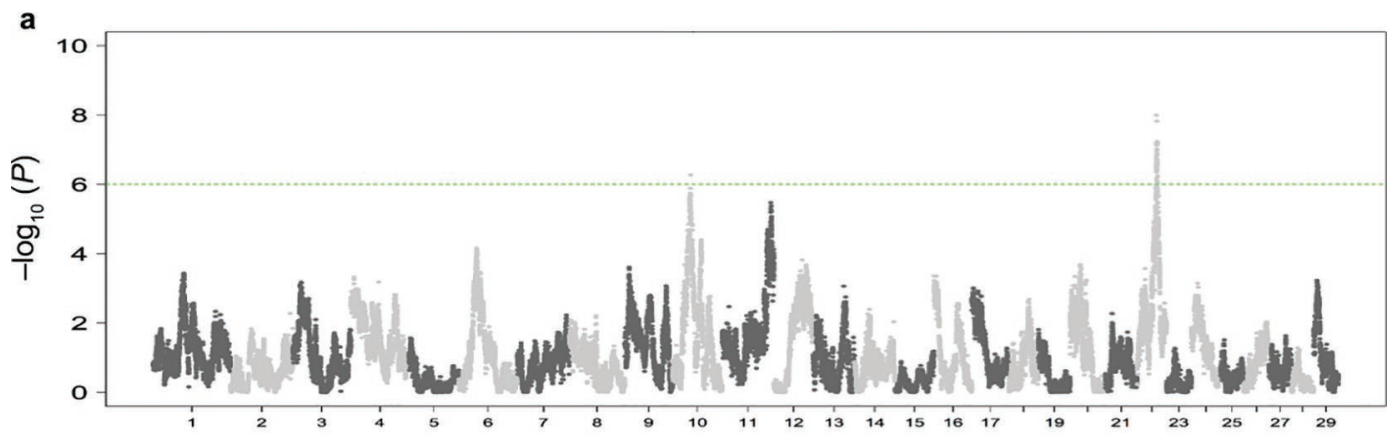

b

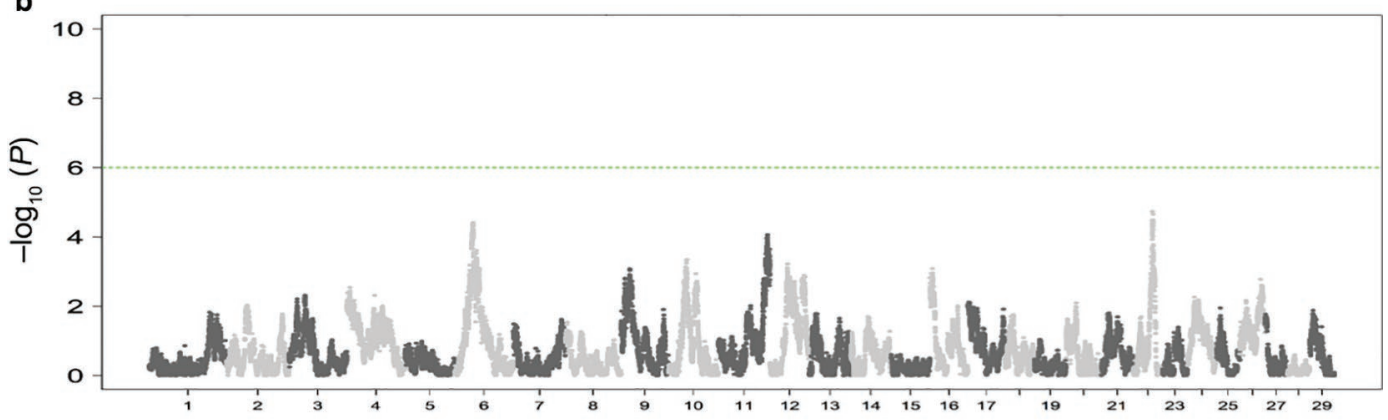

C

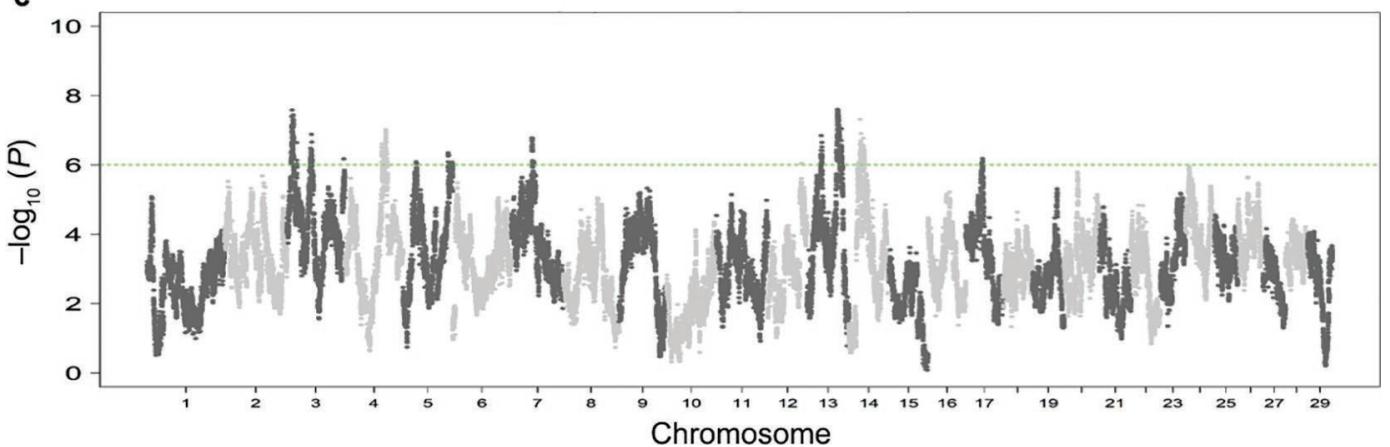

Figure 2. Genome-wide mapping of breed dominance for $(\mathrm{a})$ volume $(\mathrm{mL})$, (b) transformed number of spermatozoa $\left(\times 10^{9} / \mathrm{mL}\right)$, and $(\mathrm{c})$ live spermatozoa (\%). The horizontal dotted line indicates a $-\log P$-value of 6 as the significance threshold. 

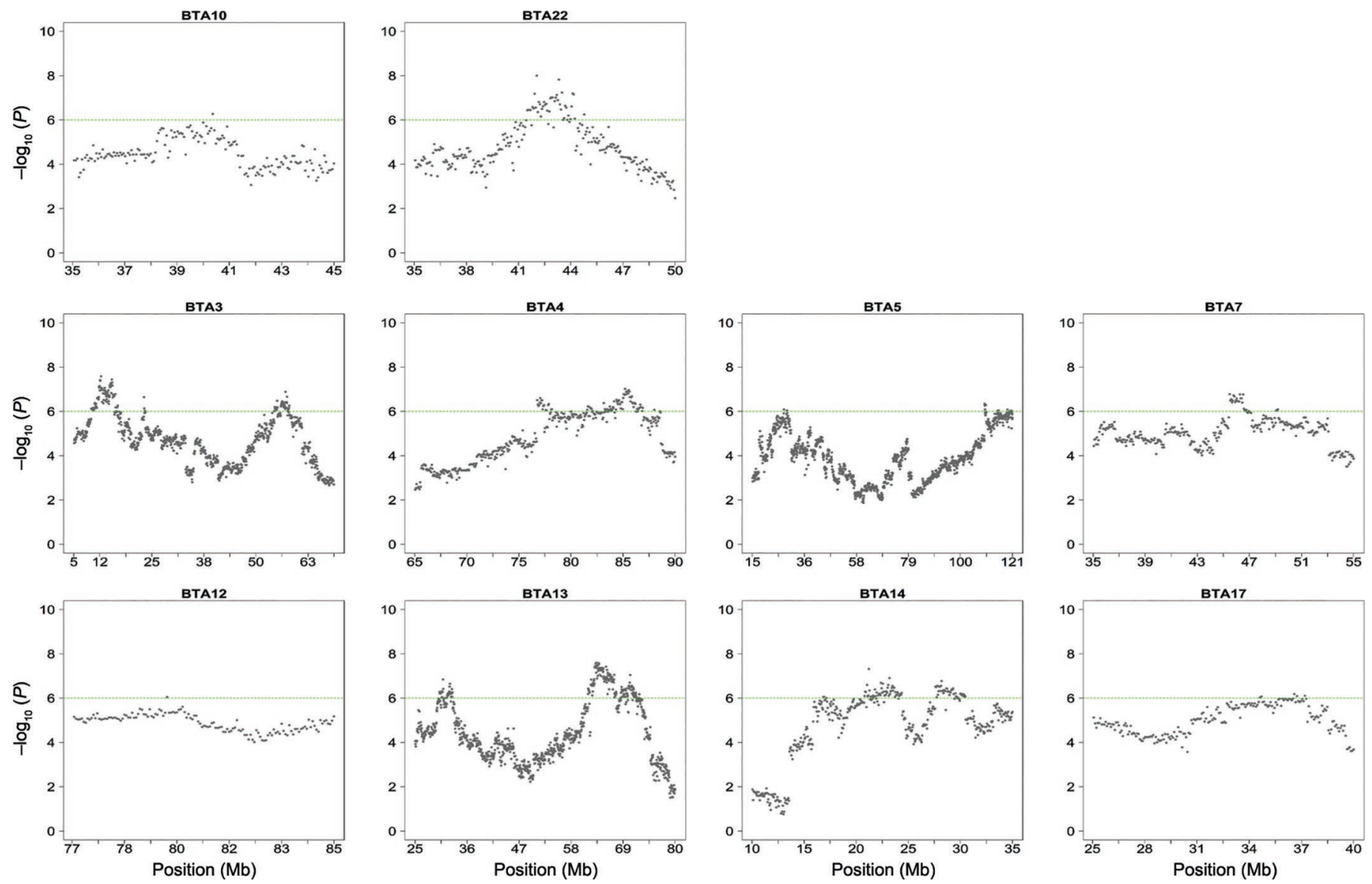

Figure 3. Significant candidate regions related to breed dominance zoomed out for volume $(\mathrm{mL})$ on BTA10 and 22 and live spermatozoa (\%) on BTA3, 4, 5, 7, 12, 13, 14, and 17. The horizontal dotted line indicates a - $\log P$-value of 6 as the significance threshold.

(43.80-45.44 and 47.31-47.39 Mb), with 5 genes in the spanning regions.

On BTA12, only 1 significant SNP was identified at $79.52 \mathrm{Mb}$, and we searched $\pm 1 \mathrm{Mb}$ for genes around this SNP. Two other wide regions $(31.36-34.62$ and 63.09-73.52 $\mathrm{Mb})$ on BTA13 and 3 significant regions (16.18-16.44, 20.08-23.95, and 27.57-29.99 Mb) on BTA14, associated with heterosis, were identified. A significant signal for heterosis on BTA17 (33.42-35.44 $\mathrm{Mb}$ ) was also detected.

\section{DISCUSSION}

In this study, we used genome-wide heterosis mapping to investigate whether specific genomic areas contributing to breed dominance, as the important component of heterosis, are associated with spermatogenesis in admixed Swiss Fleckvieh bulls. For this reason, we performed single SNP regression analysis and identified 19 regions, covering a total of 552 genes. In the following, we report only the genes related to spermatogenesis, male fertility, and semen quality with known function in Entrez, UniProt, or GeneCard. The list of all genes with their main functions is presented in Supplemental Table S1 (https://doi.org/10.3168/jds.2019-16899).

\section{Volume}

On BTA10 for volume (mL), the MDGA2 (39.82$40.75 \mathrm{Mb})$ and RPL10L $(39.64-39.65 \mathrm{Mb})$ genes were found. The MDGA2 gene is recognized to be in protein metabolism and cell-cell interactions, and the RPL10L gene encodes ribosomal proteins with a role in compensating for the inactivated X-linked gene during spermatogenesis. The DNAH12 (43.64-43.83 Mb) gene with ATPase activity BTA22 was associated with sperm motility, expressed in testis cells in intracellular movement of the organelles and vesicles. The $P D H B$ (42.91-42.93 Mb), PDE12 (43.63-43.64 Mb), and PXK (42.92-43.01 Mb) genes with ATPase activity and the ARHGEF3 (44.017-44.33 Mb) gene with GTPase activity, located in this region, play important roles in energy metabolism and oxidative phosphorylation in mitochondria and the cell membrane. 
$\% L S$

The ATP1A4 (9.48-9.53 Mb) gene was detected in the first region of BTA3 (9.34-16.45 Mb), associated with ATP and $\mathrm{Na}^{+}-\mathrm{K}^{+}$transportation in plasma membrane, with role in sperm motility and HDGF (14.08-14.09 $\mathrm{Mb})$, which is expressed in the testis with cell division and proliferation functions. On the second signal on BTA3 (24.56-24.75 Mb), the sperm-associated antigen 17 (SPAG17) with a role in sperm motility and male fertility was detected. The SPATA1 gene (59.52-59.59 $\mathrm{Mb}$ ), which seems to regulate spermatogenesis, was identified on the third signal on BTA3 (59.35-62.44 $\mathrm{Mb})$. At the end of BTA3, 1 significant SNP (119.07 $\mathrm{Mb})$ was detected, where the GPC1 (119.86-119.89 $\mathrm{Mb})$ and COPS9 (119.66-119.67 Mb) genes were found to be responsible for cell division and cell proliferation. The NDUFA10 (118.88-118.92 Mb) gene with NADH dehydrogenase activity and $A Q P 12 B$ (120.02-120.03 $\mathrm{Mb})$ in cell membrane transportation are important in energy metabolism.

Two positive signals were identified on BTA4 and SPAM1 (88.201-88.22 Mb), which is involved in fertility and sperm-egg adhesion by hyaluronidase activity in the zona pellucida. Moreover, NDUFA5 (87.869-87.89 $\mathrm{Mb}$ ) was found with NADH dehydrogenase activity in the mitochondrial respiratory chain, which is important in metabolism.

Among 8 genes related to male fertility on chromosome 5, only 1 gene (SPATS2) associated with spermatogenesis was found on the first signal (28.85-30.19 $\mathrm{Mb})$. The ACR (120.013-120.02 Mb), TSPO (113.928$113.94 \mathrm{Mb})$, SYCE3 (119.854-119.88 Mb), EFCAB6 (114.258-114.51 Mb), NUP50 (115.588-115.61 Mb), PKDREJ (116.531-116.54 Mb), and ODF3B (119.828$119.83 \mathrm{Mb}$ ) genes were identified on the another signal on chromosome 5 (113.34-120.75 Mb). The TSPO gene has a precise physiological role in lipid metabolism and steroid hormone biosynthesis. The EFCAB6 gene is linked to spermatogenesis by involvement in the androgen signaling pathway, and NUP50 is primarily expressed in the testis and is related to cell cycle and meiosis in differentiation of spermatocytes. Acrosin $(A C R)$ has a major proteinase function in the acrosome of mature spermatozoa, which facilitate the penetration of sperm through the glycoprotein layer of the ovum in the zona pellucida. The PKDREJ gene was also identified in this relatively wide signal on chromosome 5, exclusively expressed in the testis with a main role in reproduction. It is directly involved in initiating the acrosome reaction of the sperm to egg jelly during fertilization. The ODF3B gene is an important gene with a function in the outer dense fiber of the spermatozoa tail. The SYCE3 gene, expressed in the testis, is a ma- jor component in forming the homologous chromosome during protease meiosis and is implicitly required for sperm fertilization.

The DAZAP1 (43.82-43.84 Mb), ODF3L2 (43.14$43.15 \mathrm{Mb})$, THEG (43.06-43.08 Mb), and ATP8B3 (44.12-44.13 Mb) genes were located on the first signal and the CATSPER3 (46.42-46.46 Mb) gene was located on the second signal on BTA7, related to male fertility. The DAZAP1 gene has a substantial role in testes development and spermatogenesis. The $D A Z$ gene in the $Y$ chromosome arose from transportation, amplification, and pruning of the autosomal $D A Z L$ gene, which is deleted from the $\mathrm{Y}$ chromosome in infertile males with azoospermia. The ODF3L2 and THEG genes are expressed in the outer dense fiber of spermatozoa and in the nucleus of the male germ cell during spermatogenesis, respectively. The ATP8B3 gene is responsible for the maintenance of asymmetric distribution of phosphatidylserine in spermatozoa membranes and is involved in acrosome reactions and binding of spermatozoa to the zona pellucida.

The CATSPER 3 gene was found on the second signal on BTA7, related to voltage-gated calcium channel, and plays a central role in calcium-dependent physiological responses. This is essential for successful fertilization, such as sperm hyperactivation, acrosome reaction, and chemotaxis toward the oocyte. The TEX30 gene $(79.00-79.02 \mathrm{Mb})$ is expressed in the testis and was found on BTA12.

The SPAG4 (64.84-64.85 Mb), CNBD2 (65.1-65.15 $\mathrm{Mb}), P K I G(72.948-73.03 \mathrm{Mb})$, and ADA (73.029-73.05 $\mathrm{Mb})$ genes spanned along the second region of BTA13. The mammalian sperm flagellum contains cytoskeletal structure, surrounding the outer dense fiber, and $S P A G_{4}$ is required for this formation in the sperm tail, increasing sperm motility and fertilization. The CNBD2 gene is essential for male fertility and spermatogenesis, regulating sperm motility by controlling the development of the flagellar bending of sperm. The $P K I G$ gene is highly expressed in testis tissue with protein kinase activity, and $A D A$ is related to male fertility and has a protective role in early embryonic implantation.

No gene was identified to be directly associated with male fertility and spermatogenesis on BTA14. The SPATA5 (34.42-34.75 Mb) gene, responsible for maintenance of mitochondria morphology and function during spermatogenesis, and ADAD1 (35.22-35.31 Mb), in testis tissue and with a role in spermatogenesis, were found on a positive signal on BTA17 (33.42-35.44 Mb).

In the Ferenčaković et al. (2017) study, negative signals were identified for inbreeding depression associated with TNS on BTA10, 17, 20, and 27 and with \%LS on BTA1, 6, 10, and 14. We did not find any overlap between the signals that were detected by our study 
for breed dominance in admixed Swiss Fleckvieh bulls and those signals, identified for inbreeding depression in Austrian Fleckvieh bulls. The signals that we found were quite wider than the Ferenčaković et al. (2017) inbreeding depression signals, which is due to recent admixture in Swiss Fleckvieh composites compared with more ancient inbreeding in the Austrian Fleckvieh.

\section{CONCLUSIONS}

We aimed to perform genome-wide mapping of breed dominance for semen traits in admixed Swiss Fleckvieh bulls. Our definition of breed dominance considered only the ancestry origin of SNP and not whether an SNP is homozygous or heterozygous according to the straight genotype information. We found relatively wide signals for breed dominance related to volume $(\mathrm{mL})$ and \%LS caused by the comparatively small number of recombination events in this recent composite. Yet genes involved in spermatogenesis were found there, and these are strong candidates for detailed study of their function in the purebred as well as admixed populations. Further study is needed to map epistatic interaction, as the other important component of heterosis, and to perform appropriate simulation strategies to decide the most appropriate source for coding the heterosis components (straight genotypes or breed origin of these markers).

\section{ACKNOWLEDGMENTS}

We thank the Swissherdbook cooperative Zollikofen for providing genotypes and Swissgenetics for providing phenotypes for analyses. The phenotypic and genotypic data analyzed in this study were obtained from Swissgenetics (Zollikofen, Switzerland) and Swissherdbook Cooperation (Zollikofen, Switzerland). Requests for access to the phenotypes and genotypes to facilitate replication of the published results can be addressed to Swissgenetics and Swissherdbook Cooperative (Zollikofen, Switzerland). Y. T. Utsunomiya was supported by Sao Paulo Research Foundation-FAPESP (Sao Paulo, Brazil; process 2014/01095-8 and 2016/07531-0).

\section{REFERENCES}

Akanno, E. C., M. K. Abo-Ismail, L. H. Chen, J. J. Crowley, Z. Q. Wang, C. X. Li, J. A. Basarab, M. D. MacNeil, and G. S. Plastow. 2018a. Modeling heterotic effects in beef cattle using genome-wide SNP-marker genotypes. J. Anim. Sci. 96:830-845.

Akanno, E. C., L. Chen, M. K. Abo-Ismail, J. J. Crowley, Z. Wang, C. Li, J. A. Basarab, M. D. MacNeil, and G. Plastow. 2017. Ge- nomic prediction of breed composition and heterosis effects in Angus, Charolais, and Hereford crosses using 50K genotypes. Can. J. Anim. Sci. 97:431-438.

Akanno, E. C., L. H. Chen, M. K. Abo-Ismail, J. J. Crowley, Z. Q. Wang, C. X. Li, J. A. Basarab, M. D. MacNeil, and G. S. Plastow. 2018b. Genome-wide association scan for heterotic quantitative trait loci in multi-breed and crossbred beef cattle. Genet. Sel. Evol. 50:48.

Alexander, D. H., J. Novembre, and K. Lange. 2009. Fast model-based estimation of ancestry in unrelated individuals. Genome Res. 19:1655-1664.

Basarab, J. A., J. J. Crowley, M. K. Abo-Ismail, G. M. Manafiazar, E. C. Akanno, V. S. Baron, and G. Plastow. 2018. Genomic retained heterosis effects on fertility and lifetime productivity in beef heifers. Can. J. Anim. Sci. 98:642-655.

Box, G. E. P., and D. R. Cox. 1964. An analysis of transformations. J R. Stat. Soc. B 26:211-252.

Chang, C. C., C. C. Chow, L. C. A. M. Tellier, S. Vattikuti, S. M. Purcell, and J. J. Lee. 2015. Second-generation PLINK: Rising to the challenge of larger and richer datasets. Gigascience 4:7.

Cundiff, L. V. 1970. Experimental results on crossbreeding cattle for beef production. J. Anim. Sci. 30:694-705.

Dickerson, G. E. 1973. Inbreeding and heterosis in animals. J. Anim. Sci. 1973(Symp.):54-77.

Falconer, D. S., and T. F. C. Mackay. 1996. Introduction to Quantitative Genetics. 4th ed. Longman, Harlow, UK.

Ferenčaković, M., J. Solkner, M. Kaps, and I. Curik. 2017. Genomewide mapping and estimation of inbreeding depression of semen quality traits in a cattle population. J. Dairy Sci. 100:4721-4730.

Freyer, G., S. Konig, B. Fischer, U. Bergfeld, and B. G. Cassell. 2008. Invited review: Crossbreeding in dairy cattle from a German perspective of the past and today. J. Dairy Sci. 91:3725-3743.

Gregory, K. E., L. A. Swiger, L. J. Sumption, R. M. Koch, J. E. Ingalls, W. W. Rowden, and J. A. Rothlisberger. 1966. Heterosis effects on growth rate and feed efficiency of beef steers. J. Anim. Sci. 25:299-310.

Hill, W. G. 1982. Dominance and epistasis as components of heterosis. Z. Tierzuecht. Zuechtungsbiol. 99:161-168.

Kargo, M., P. Madsen, and E. Norberg. 2012. Short communication: Is crossbreeding only beneficial in herds with low management level? J. Dairy Sci. 95:925-928.

Khayatzadeh, N., G. Mészáros, M. Ferenčaković, Y. T. Utsunomiya, U. Schnyder, F. Seefried, I. Curik, and J. Sölkner. 2018a. Genomewide mapping of dominance component of heterosis for percentage of live sperm in admixed Swiss Fleckvieh bulls. Page 577 in Proc. World Congress on Genetics Applied to Livestock Production (WCGALP), Auckland, New Zealand. Volume Electronic Poster Session-Methods and Tools-Models and Computing Strategies 2.

Khayatzadeh, N., G. Meszaros, Y. T. Utsunomiya, F. Schmitz-Hsu, F. Seefried, U. Schnyder, M. Ferencakovic, J. F. Garcia, I. Curik, and J. Solkner. 2018b. Effects of breed proportion and components of heterosis for semen traits in a composite cattle breed. J. Anim. Breed. Genet. 135:45-53.

Kinghorn, B. 1983. Genetic effects in crossbreeding. Z. Tierzuecht Zuechtungsbiol. 100:209-222.

Purcell, S., B. Neale, K. Todd-Brown, L. Thomas, M. A. R. Ferreira, D. Bender, J. Maller, P. Sklar, P. I. W. de Bakker, M. J. Daly, and P. C. Sham. 2007. PLINK: A tool set for whole-genome association and population-based linkage analyses. Am. J. Hum. Genet. $81: 559-575$.

Sankararaman, S., S. Sridhar, G. Kimmel, and E. Halperin. 2008 Estimating local ancestry in admixed populations. Am. J. Hum. Genet. 82:290-303.

SAS Institute Inc. 2009. SAS/STAT 9.2 User's Guide. SAS Inst. Inc., Cary, NC.

Shull, G. H. 1908. The composition of a field of maize. J. Heredity os $-4: 296-301$. 